\title{
Initial Pose Estimation for 3D Model Tracking Using Learned Objective Functions
}

\author{
Matthias Wimmer*1 and Bernd Radig ${ }^{2}$ \\ ${ }^{1}$ Faculty of Science and Engineering, Waseda University, Tokyo, Japan \\ ${ }^{2}$ Institut für Informatik, Technische Universität München, Germany
}

\begin{abstract}
Tracking 3D models in image sequences essentially requires determining their initial position and orientation. Our previous work [14] identifies the objective function as a crucial component for fitting 2D models to images. We state preferable properties of these functions and we propose to learn such a function from annotated example images.

This paper extends this approach by making it appropriate to also fit 3D models to images. The correctly fitted model represents the initial pose for model tracking. However, this extension induces nontrivial challenges such as out-of-plane rotations and self occlusion, which cause large variation to the model's surface visible in the image.

We solve this issue by connecting the input features of the objective function directly to the model. Furthermore, sequentially executing objective functions specifically learned for different displacements from the correct positions yields highly accurate objective values.
\end{abstract}

\section{Introduction}

Model-based image interpretation is appropriate to extract high-level information from single images and from image sequences. Models induce a priori knowledge about the object of interest and thereby reduce the large amount of image data to a small number of model parameters. However, the great challenge is to determine the model parameters that best match a given image. For interpreting image sequences, model tracking algorithms fit the model to the individual images of the sequence. Each fitting step benefits from the pose estimate derived from the previous image of the sequence. However, determining the pose estimate for the first image of the sequence has not been sufficiently solved yet. The challenge of this so-called initial pose estimation is identical to the challenge of fitting models to single images.

Our previous work identifies the objective function as an essential component fitting models to single images [14]. This function evaluates how well a particular model fits to an image. Without losing generality, we consider lower values to represent a better model fitness. Therefore, algorithms search for the model parameters that minimize the objective function. Since the described methods

\footnotetext{
* This research is partly funded by a JSPS Postdoctoral Fellowship for North American and European Researchers (FY2007).
} 
are independent of the used fitting algorithm, we do not elaborate on them, but refer to Hanek et al. [5] for a recent overview and categorization of fitting algorithms. As our approach has only been specified for 2D models so far, this paper extends it to be capable for handling 3D models while considering a rigid model of a human face. In contrast to artificial objects, such as cars, faces highly vary in shape and texture and therefore the described face model fitting task represents a particular difficulty.

Many researchers engage in fitting 3D models. Lepetit et al. [7] treat this issue as a classification problem and use decision trees for solution. As an example implementation, the ICP algorithm $[2,12]$ minimizes the square error of the distance between the visible object and the model projected to the image.

Problem Statement. Although the accuracy of model fitting heavily depends on the objective function, it is often designed by hand using the designer's intuition about a reasonable measure of fitness. Afterwards, its appropriateness is subjectively determined by inspecting the objective function on example images and example model parameters. If the result is not satisfactory the function is tuned or redesigned from scratch [11,4], see Figure 1 (left). Therefore, building the objective function is very time consuming and the function does not guarantee to yield accurate results.
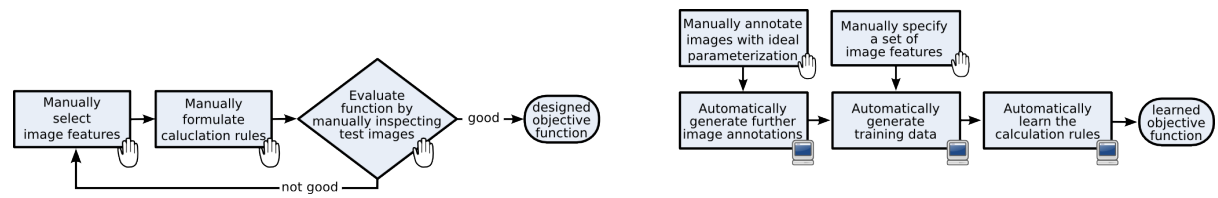

Fig. 1. The procedures for designing (left) and learning (right) objective functions.

Solution Outline. Our novel approach focuses on the root problem of model fitting: We improve the objective function rather than the fitting algorithm. As a solution to this challenge, we propose to conduct a five-step methodology that learns robust local objective functions from annotated example images. We investigated this approach for 2D models so far [14]. This paper extends our methodology in order to generate objective functions that are capable of handling 3D models as well. The obtained functions consider specific aspects of 3D models, such as out-of-plane rotations and self-occlusion. We compute the features not in the 2D image plane but in the space of the $3 \mathrm{D}$ model. This requires connecting the individual features directly to the model.

Contributions. The resulting objective functions work very accurately in real-world scenarios and they are able to solve the challenge of initial pose estimation that is required by model tracking. This easy-to-use approach is applicable to various image interpretation scenarios and requires the designer just to annotate example images with the correct model parameters. Since no further computer vision expertise is necessary, this approach has great potential for commercialization. 
The paper proceeds as follows. In Section 2, we sketch the challenge of modelbased image interpretation. In Section 3, we propose our methodology to learn accurate local objective functions from annotated training images with particular focus on 3D models. Section 4 conducts experimental evaluations that verify this approach. Section 5 summarizes our approach and shows future work.

\section{Model-based Image Interpretation}

Rigid 3D models represent the geometric properties of real-world objects. A six-dimensional parameter vector $\boldsymbol{p}=\left(t_{x}, t_{y}, t_{z}, \alpha, \beta, \gamma\right)^{T}$ describes the position and orientation. The model consists of $1 \leq n \leq N$ three-dimensional model points specified by $\boldsymbol{c}_{n}(\boldsymbol{p})$. Figure 2 depicts our face model with $N=214$ model points.

Fitting 3D models to images requires two essential components: The fitting algorithm searches for the model parameters that match the content of the image best. For this task, it searches for the minimum of the objective function $f(I, \boldsymbol{p})$, which determines how well a model $\boldsymbol{p}$ matches an image $I$. As in Equation 1, this function is often subdivided into $N$ local components $f_{n}(I, \boldsymbol{x})$, one for each model point $[7,1,9]$. These local functions determine how well the $n^{\text {th }}$ model point fits to the image. The advantage of this partitioning is that designing the local functions is more straightforward than designing the global function, because only the image content in the vicinity of one projected model point needs to be taken into consideration. The disadvantage is that dependencies and interactions between local errors cannot be combined.

$$
f(I, \boldsymbol{p})=\sum_{n=1}^{N} f_{n}\left(I, \boldsymbol{c}_{n}(\boldsymbol{p})\right)
$$

\subsection{Characteristic Search Directions of Local Objective Functions}

Fitting algorithms for 2D contour models usually search the minimum of the objective function along the perpendicular to the contour [3]. The objective function computes its value from image features in the vicinity of the perpendicular. We stick to this procedure, and therefore we create local objective functions that are
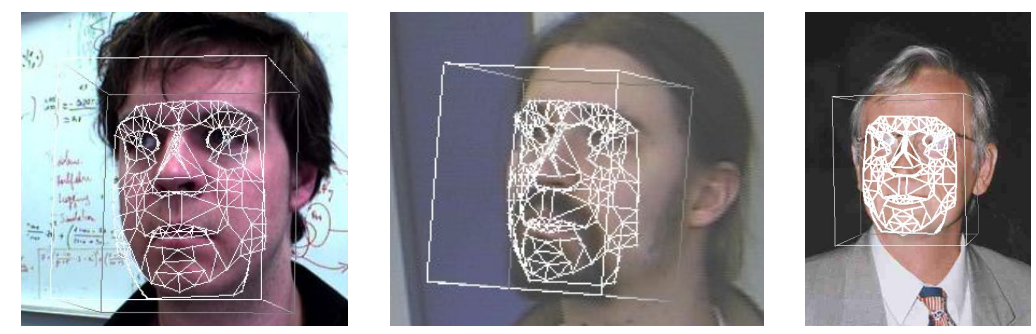

Fig. 2. Our 3D model of a human face correctly fitted to images. 

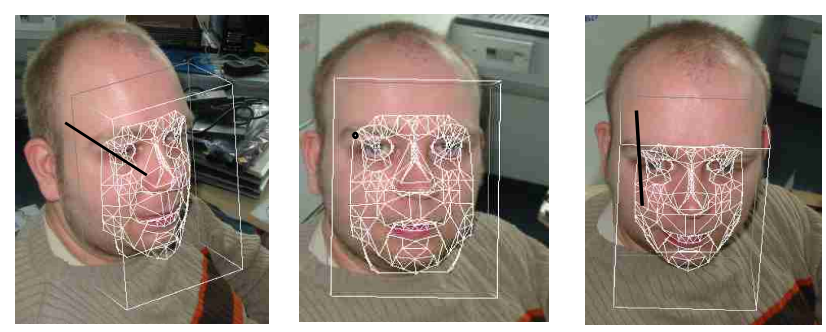

Fig. 3. Due to affine transformations of the face model the characteristic search directions will not be parallel to the image plane, in general. These three images show how the transformations affect one of these directions.

specific to a search direction. These so-called characteristic directions represent three-dimensional lines and we connect them tightly to the model's geometric structure, i.e. to the individual model points $\boldsymbol{c}_{n}(\boldsymbol{p})$. Transforming the model's pose transforms these directions equivalently, see Figure 3. The objective function computes its value from three-dimensional features, however their value is calculated projecting them to the image plane.

An image is most descriptive for a characteristic direction if they are parallel. Unfortunately, transforming the model will usually yield characteristic directions that are not parallel to the image plane. Therefore, we consider not only one but $1 \leq l \leq L$ characteristic directions per model point, which are differently oriented. These directions may be arbitrary, but we prefer them to be pairwise orthogonal. This yields $L$ objective function $f_{n, l}(I, \boldsymbol{x})$ for each model point. In order not to increase computation time, we consider the characteristic direction, which is most parallel to the image, only.

$$
f_{n}(I, \boldsymbol{x})=f_{n, g_{n}(p)}(I, \boldsymbol{x})
$$

The model point's local objective function $f_{n}$ is computed as in Equation 2. The indicator $g_{n}(\boldsymbol{p})$ computes the index of the characteristic direction that is most significant for the current pose $\boldsymbol{p}$ of the model, i.e. that is most parallel to the image plane.

\section{Learning Objective Functions from Image Annotations}

Ideally, local objective functions have two specific properties. First, they should have a global minimum that corresponds to the best model fit. Otherwise, we cannot be certain that determining the true minimum of the local objective function indicates the intended result. Second, they should have no other local minima. This implies that any minimum found corresponds to the global minimum, which facilitates search. A concrete example of an ideal local objective function, that has both properties, is shown in Equation 3. $\boldsymbol{p}_{I}^{\star}$ denotes the model parameters with the best model fit for a certain image $I$. 


$$
f_{n}^{\star}(I, \boldsymbol{x})=\left|\boldsymbol{x}-\boldsymbol{c}_{n}\left(\boldsymbol{p}_{I}^{\star}\right)\right|
$$

Unfortunately, $f_{n}^{\star}$ cannot be applied to unseen images, for which the best model parameters $\boldsymbol{p}_{I}^{\star}$ are not known. Nevertheless, we apply this ideal objective function to annotated training images and obtain ideal training data for learning a local objective function $f_{n, l}^{\ell}$ for the model point $n$ and its characteristic direction $l$. The key idea behind our approach is that since the training data is generated by an ideal objective function, the learned function will also be approximately ideal. This has already been shown in [14]. Figure 1 (right) illustrates the proposed five-step procedure.

Step 1: Annotating Images with Ideal Model Parameters. As in Figure 2, a database of $1 \leq k \leq K$ images $I_{k}$ is manually annotated with the ideal model parameters $\boldsymbol{p}_{I_{k}}^{\star}$, which are necessary to compute the ideal objective functions $f_{n}^{\star}$. This is the only laborious step of the entire procedure.

Step 2: Generating Further Image Annotations. The ideal objective function returns the minimum $f_{n}^{\star}(I, \boldsymbol{x})=0$ for all manual annotations $\boldsymbol{x}=\boldsymbol{c}_{n}\left(\boldsymbol{p}_{I_{k}}^{\star}\right)$.

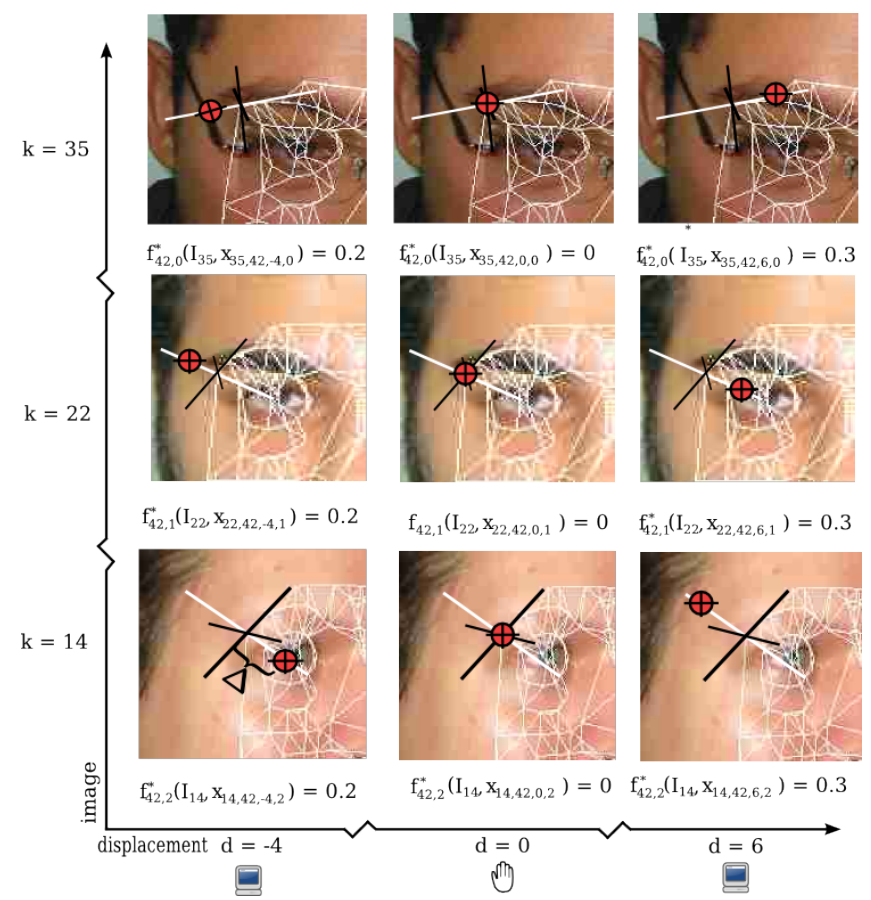

Fig. 4. Further annotations are generated by moving along the line that is longest when projected in the image. That line is colored white here. The directions illustrated in black are not used. Annotations on one of the directions that are not used are also shown to demonstrate that this direction is too short to be used. 


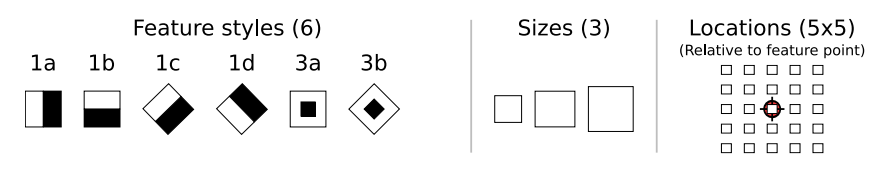

Fig. 5. This comprehensive set of image features is provided for learning local objective functions. In our experiments, we use a total number of $A=6 \cdot 3 \cdot 5 \cdot 5=450$ features.

These annotations are not sufficient to learn the characteristics of $f_{n}^{\star}$. Therefore, we will generate annotations $\boldsymbol{x} \neq \boldsymbol{c}_{n}\left(\boldsymbol{p}_{I_{k}}^{\star}\right)$, for which $f_{n}^{\star}(I, \boldsymbol{x}) \neq 0$. In general, any $3 \mathrm{D}$ position $\boldsymbol{x}$ may represent one of these annotations, however, we sample $-D \leq d \leq D$ positions along the characteristic direction with a maximum displacement $\Delta$ (learning radius), see Figure 4 . This procedure learns the calculation rules of the objective function more accurately. In this paper, we use $L=3$ characteristic directions, because the model points vary within the $3 \mathrm{D}$ space. Note that the $g_{n}\left(\boldsymbol{p}_{I_{k}}^{\star}\right)$ selects the most significant direction for the $n^{\text {th }}$ model point.

Step 3: Specifying Image Features. Our approach learns the calculation rules of a mapping $f_{n, l}^{\ell}$ from an image $I_{k}$ and a location $\boldsymbol{x}_{k, n, d, l}$ to the value of $f_{n}^{\star}\left(I_{k}, \boldsymbol{x}_{k, n, d, l}\right)$. Since $f_{n, l}^{\ell}$ has no knowledge of $\boldsymbol{p}_{I}^{\star}$, it must compute its result from the image content. Instead of learning a direct mapping from the pixel values in the vicinity of $\boldsymbol{x}$ to $f_{n}^{\star}$, we compute image features, first. Note that $\boldsymbol{x}$ does not denote a position in $I$ but in 3D space. However, the corresponding pixel position is obtained via perspective projection.

Our idea is to provide a multitude of $1 \leq a \leq A$ features, and let the training algorithm choose which of them are relevant to the calculation rules of $f_{n, l}^{\ell}$. Each feature $h_{a}(I, \boldsymbol{x})$ is computed from an image $I$ and a position $\boldsymbol{x}$ and delivers a scalar value. Our approach currently relies on Haar-like features $[13,8]$ of different styles and sizes. Furthermore, the features are not only computed at the location of the model point itself, but also at positions on a grid within its vicinity, see Figure 5. This variety of styles, sizes, and locations yields a set of $A=450$ image features as we use it in our experiments in Section 4. This

$d=-4$

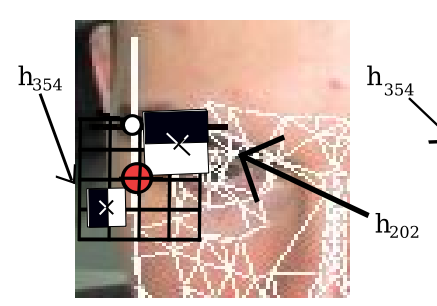

$\mathrm{f}_{41}^{*}\left(\mathrm{I}_{22}, \mathrm{x}_{22,41,-4,1}\right)=0.2$ $\mathrm{d}=0$

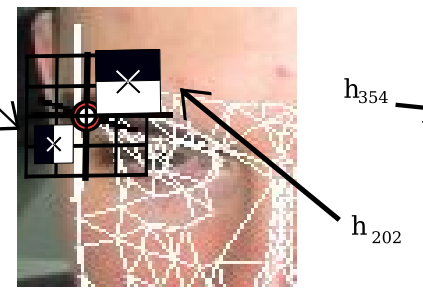

$\mathrm{f}_{41}^{*}\left(\mathrm{I}_{22}, \mathrm{x}_{22,41,0,1}\right)=0$ $d=6$

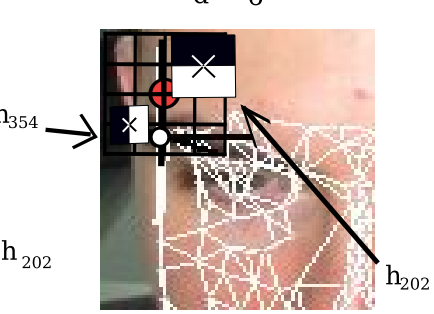

$\mathrm{f}_{41}^{*}\left(\mathrm{I}_{22}, \mathrm{x}_{22,41,6,1}\right)=0.3$

Fig. 6. The grid of image features moves along with the displacement. 
multitude of features enables the learned objective function to exploit the texture of the image at the model point and in its surrounding area. When moving the model point, the image features move along with it, leading their values to change, see Figure 6 .

Step 4: Generating Training Data. The result of the manual annotation step (Step 1) and the automated annotation step (Step 2) is a list of correspondences between positions $\boldsymbol{x}$ and the corresponding value of $f_{n}^{\star}$. Since $K$ images, $N$ model points, and $2 D+1$ displacements are landmarked these correspondences amount to $K \cdot N \cdot(2 D+1)$. Equation 4 illustrates the list of these correspondences.

$$
\begin{aligned}
& {\left[\begin{array}{lll}
I_{k}, & \boldsymbol{x}_{k, n, d, l}, & \left.f_{n}^{\star}\left(I_{k}, \boldsymbol{x}_{k, n, d, l}\right)\right]
\end{array}\right.} \\
& {\left[\boldsymbol{h}_{1}\left(I_{k}, \boldsymbol{x}_{k, n, d, l}\right), \ldots, \boldsymbol{h}_{A}\left(I_{k}, \boldsymbol{x}_{k, n, d, l}\right), f_{n}^{\star}\left(I_{k}, \boldsymbol{x}_{k, n, d, l}\right)\right]} \\
& \text { with } 1 \leq k \leq K, 1 \leq n \leq N,-D \leq d \leq D, l=g_{n}\left(p_{I_{k}}\right)
\end{aligned}
$$

Applying the list of image features to the list of correspondences yields the training data in Equation 5. This step simplifies matters greatly. Since each feature returns a single value, we hereby reduce the problem of mapping the vast amount of image data and the related pixel locations to the corresponding target value, to mapping a list of feature values to the target value.

Step 5: Learning the Calculation Rules. Given the training data from Equation 5 , the goal is to now learn the function $f_{n, l}^{\ell}(I, \boldsymbol{x})$ that approximates $f_{n}^{\star}(I, \boldsymbol{x})$. The challenge is that $f_{n, l}^{\ell}$ is not provided knowledge of $\boldsymbol{p}_{I}^{\star}$. Therefore, it can be applied to previously unseen images. We obtain this function by training a model tree $[10,15]$ with the comprehensive training data from Equation 5. Note that the $N \cdot L$ objective functions $f_{n, l}^{\ell}$ have to be learned individually. However, learning $f_{n, l}^{\ell}$ only requires the records of the training data (Equation 5) where $n$ and $l$ match.

Model trees are a generalization of regression trees and, in turn, of decision trees. Whereas decision trees have nominal values at their leaf nodes, model trees have line segments, allowing them to also map features to a continuous value, such as the value returned by the ideal objective function. One of the reasons for deciding for model trees is that they tend to select only features that are relevant to predict the target value. Therefore, they pick a small number of $M_{n}$ Haar-like features from the provided set of $A \gg M_{n}$ features.

After executing these five steps, we obtain a local objective function $f_{n, l}^{\ell}$ for each model point $n$ and each direction $l$. It can now be called with an arbitrary location $\boldsymbol{x}$ of an arbitrary image $I$. The learned model tree calculates the values of the necessary features from the image content and yields the result value.

\section{Experimental Evaluation}

In this section, two experiments show the capability of fitting algorithms equipped with a learned objective function to fit a face model to previously unseen images. The experiments are performed on 240 training images and 80 test images 


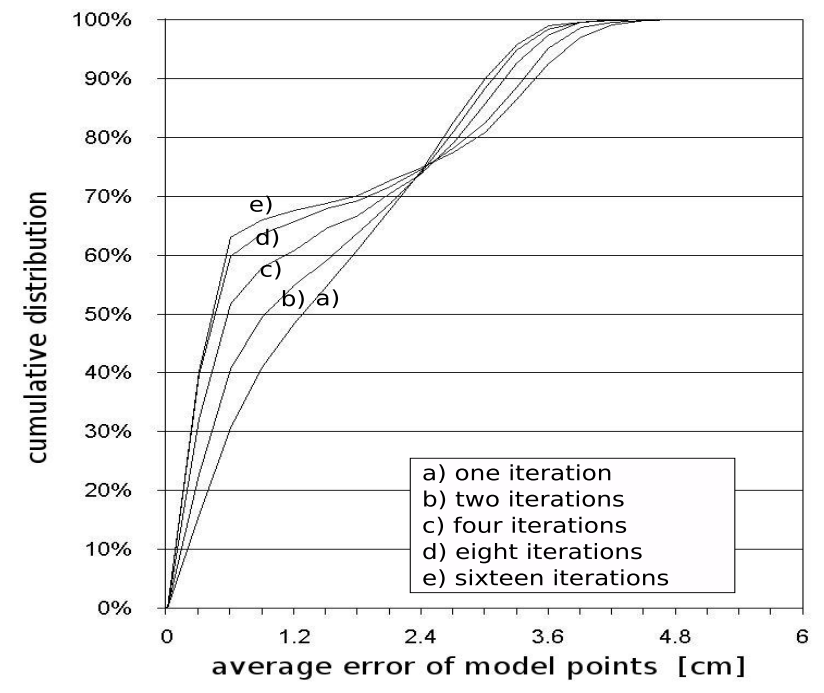

Fig. 7. If the initial distance to the ideal model points is smaller than the learning radius this distance is further reduced with every iteration. Otherwise, the result of the fitting step is unpredictable and the model points are spread further.

with a non-overlapping set of individuals. Furthermore, the images differ in face pose, illumination, and background. Our evaluations randomly displace the face models from the manually specified pose. The fitting process determines the position of every model point by exhaustively searching along the most significant characteristic direction for the global minimum of the local objective function. Afterwards, the model parameters $\boldsymbol{p}$ are approximated. The subsequent figures illustrate the average point-to-point error of the model points between the obtained model $\boldsymbol{p}$ and the manually specified model $\boldsymbol{p}_{I}^{\star}$.

Our first evaluation investigates the impact of executing the fitting process with a different number of iterations. Figure 7 illustrates that each iteration improves the model parameters. However, there is a lower bound to the quality of the obtained model fit. Obviously, more than 10 iterations do not improve the fraction of well-fitted models significantly. Note that the objective function's value is arbitrary for high distances from the correct position. These models are further distributed with every iteration.

Our second experiment conducts model fitting by subsequently applying the fitting process with two different objective functions $f^{A}$ and $f^{B}$ learned with decreasing learning radii $\Delta$. $f^{A}$ with a large $\Delta$ is able to handle large initial displacements in translation and rotation. However, the obtained fitting result gets less accurate, see Figure 8 . The opposite holds true for $f^{B}$. The idea is to apply a local objective function learned with large $\Delta$ first and then gradually apply objective functions learned with smaller values for $\Delta$. As opposed to the previous experiment, where we iteratively executed the same objective function, 


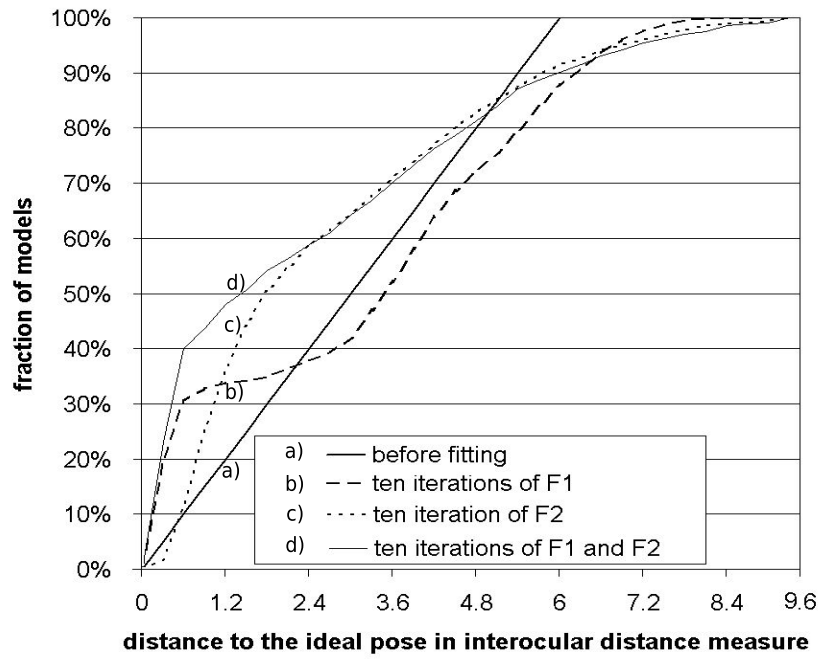

Fig. 8. By combining fitting algorithms using objective functions with different learning radius we obtain result that show the strengths of both objective functions. The sequential approach shows the tolerance to errors of $f^{A}$ and the accuracy of $f^{B}$

this iteration scheme executes different objective functions, which compensates the weakness of one function by the strength of another.

The advantage of concatenating algorithms with objective functions with decreasing learning radii compared to iterating one algorithm several times is illustrated by Figure 8. Sequentially applying $f^{A}$ and $f^{B}$ is significantly better than both of the other algorithms. Note that we execute each experiment with ten iterations, because we don't expect any improvement in quality with a higher number of iterations, see our first experiment. Therefore, the obtained accuracy from the sequential execution does not base of the fact that some additional iterations are applied.

\section{Summary and Conclusion}

In this paper, we extended our five-step methodology for learning local objective functions that we introduced for 2D models so far [14]. 3D models can now be considered as well. This approach automates many critical decisions and the remaining manual steps require little domain-dependent knowledge. Furthermore, its process does not contain any time-consuming loops. These features enable non-expert users to customize the fitting application to their specific domain.

The resulting objective function is not only able to process objects that look similarly, such as in $[7,6]$ but objects that differ significantly in shape and texture, such as human faces. Being trained with a limited number of annotated images as described in Section 3 the resulting objective function is able to fit 
faces that are not part of the training data as well. However, the database of annotated faces must be representative enough. If there were no bearded men in the training data the algorithm would have problem in fitting the model to an image of such a man. The disadvantage of our approach is the laborious annotation step. Gathering and annotating hundreds of images requires several weeks.

\section{References}

1. N. Allezard, M. Dhome, and F. Jurie. Recognition of 3D textured objects by mixing view-based and model-based representations. ICPR, pp 960-963, Sept 2000.

2. P. J. Besl and N. D. McKay. A method for registration of 3-D shapes. IEEE Transactions on Pattern Analysis and Machine Intelligence, 14(2):239-256, February 1992.

3. T. F. Cootes, C. J. Taylor, A. Lanitis, D. H. Cooper, and J. Graham. Building and using flexible models incorporating grey-level information. ICCV, pp 242-246, 1993.

4. D. Cristinacce and T. F. Cootes. Facial feature detection and tracking with automatic template selection. In $7^{\text {th }}$ IEEE International Conference on Automatic Face and Gesture Recognition, pp 429-434, April 2006.

5. R. Hanek. Fitting Parametric Curve Models to Images Using Local Self-adapting Seperation Criteria. PhD thesis, Technische Universität München, 2004.

6. V. Lepetit, P. Lagger, and P. Fua. Randomized trees for real-time keypoint recognition. In CVPR 2005, pp 775-781, Switzerland, 2005.

7. V. Lepetit, J. Pilet, and P. Fua. Point matching as a classification problem for fast and robust object pose estimation. In CVPR 2004, vol 2, pp 244-250, Jun 2004.

8. R. Lienhart and J. Maydt. An extended set of haar-like features for rapid object detection. In IEEE ICIP, pp 900-903, 2002.

9. E. Marchand, P. Bouthemy, F. Chaumette, and V. Moreau. Robust real-time visual tracking using a 2D-3D model-based approach. In ICCV, pp 262-268, Sept 1999.

10. R. Quinlan. Learning with continuous classes. In Proceedings of the $5^{\text {th }}$ Australian Joint Conference on Artificial Intelligence, pp 343-348, 1992.

11. S. Romdhani. Face Image Analysis using a Multiple Feature Fitting Strategy. PhD thesis, University of Basel, Computer Science Department, Basel, CH, January 2005.

12. D. Simon, M. Hebert, and T. Kanade. Real-time 3-D pose estimation using a highspeed range sensor. In Proceedings of IEEE International Conference on Robotics and Automation (ICRA '94), vol 3, pp 2235-2241, May 1994.

13. P. Viola and M. Jones. Rapid object detection using a boosted cascade of simple features. In Computer Vision and Pattern Recognition (CVPR), 2001.

14. M. Wimmer, S. Pietzsch, F. Stulp, and B. Radig. Learning robust objective functions with application to face model fitting. In Proceedings of the $29^{\text {th }}$ DAGM Symposium, Heidelberg, Germany, September 2007. to appear.

15. I. H. Witten and E. Frank. Data Mining: Practical machine learning tools and techniques. Morgan Kaufmann, San Francisco, $2^{\text {nd }}$ edition, 2005. 